\title{
LINEAR BIREFRINGENCE CHANGES IN BETAINE PHOSPHITE CRYSTAL
}

\author{
B. Kosturek, J. Przeslawski and Z. Czapla
}

Institute of Experimental Physics, University of Wrocław

Cybulskiego 36, 50-205 Wrocław, Poland

(Received February 11, 1993; in final form March 30, 1993)

\begin{abstract}
Linear birefringence temperature changes are measured in the betaine phoshite crystal. The linear birefringence increments induced by antiferrodistortive, structural and ferroelectric phase transitions are analysed. Temperature derivatives of the linear birefringences are shown for comparison. Critical exponents are determined for both transitions. The antiferrodistortive phase transition is close to a tricritical one.
\end{abstract}

PACS numbers: $64.70 . \mathrm{Kb}, 78.20 . \mathrm{Fm}$

\section{Introduction}

Betaine phosphite, $\left(\mathrm{CH}_{3}\right)_{3} \mathrm{~N}^{+} \mathrm{CH}_{2} \mathrm{COO}^{-} \cdot \mathrm{H}_{3} \mathrm{PO}_{3}$, abbreviated as $\mathrm{BPI}$, belongs to a family of the addition compounds of the $\alpha$-amino acid betaine and inorganic acids. Weak intermolecular and intramolecular bonds between betaine and inorganic groups make the structure of betaine compounds sensitive to tive external forces and temperature which leads to many structural phase transitions [1]. The BPI crystal is monoclinic at room temperature (the space group: $P 2_{1} / c$ ), and has four molecules per unit cell [2]. The BPI molecule consists of the betaine molecule and an inorganic acid rest of $\mathrm{H}_{3} \mathrm{PO}_{3}$. These components are bonded by a hydrogen bond between an oxygen atom of the carboxyl group $\mathrm{COO}^{-}$and one of the hydrogen atoms of the phosphoric acid. The structure of the BPI crystal is formed of the isolated BPI molecules bonded by hydrogen bonds lying approximately parallel to the $y$-axis. The phosphite groups are bonded by these disordered hydrogen bonds and a quasi-one-dimensional chain structure is built. The high temperature structural phase transition at $T_{\mathrm{C}_{1}}=355 \mathrm{~K}$ is connected with a change of the space group from $P 2_{1} / m(Z=2)$ to $P 2_{1} / c(Z=4)$ - by an analogy to the betaine phosphate, BP crystal [3]. The low temperature ferroelectric phase transition $\left(P 2_{1} / c \Rightarrow P 2_{1}\right)$ at about $T_{\mathrm{C}_{2}}=226 \mathrm{~K}$ is of the second order $\left(P_{\mathrm{s}}\right.$ along the $b$-axis). Third anomaly, probably the phase transition into the proton glass phase, is found at about $177 \mathrm{~K}$ [4]. Linear birefringence studies are regarded 
as a very sensitive tool in investigaling the ferroic phase transitions [5]. In the series of papers Kroupa et al. [6-8] described the birefringence behaviour in some betaine compounds. There is, however, a very limited set of experimental data on the phase transitions in the BPI crystal.

\section{Experimental}

The BPI crystals were grown by controlled evaporation at a temperature of $308 \mathrm{~K}$ from an aqueous solution containing the betaine and $\mathrm{H}_{3} \mathrm{PO}_{3}$ in the molar ratio 1:1. Samples were cut perpendicularly to the directions of the optical indicatrix axes. An orientation of the optical indicatrix and the cleavage plane relative to the crystal axes is shown in Fig. 1. The refractive indices were determined at

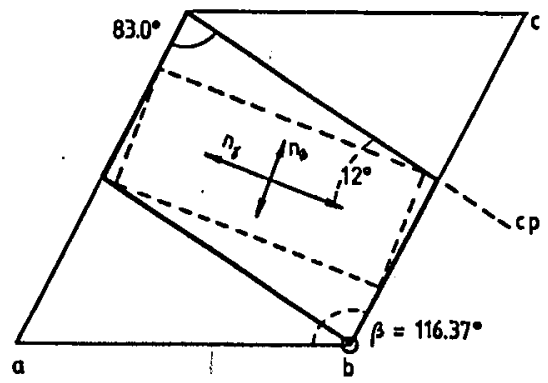

Fig. 1. Orientation of the oplical indicatrix relative to the crystal axes. Thick lines: natural faces, $c p-$ cleavage plane [4].

$\lambda=589 \mathrm{~nm}: n_{\alpha}=1.478, n_{\beta}=1.498$, and $n_{\gamma}=1.527$ [4], and the crystal can be regarded as optically positive; an angle $V=40.4^{\circ}$. The linear birefringences are denoted as: $\Delta n_{\alpha}=\left|n_{\beta}-n_{\gamma}\right|=\Delta n_{b}, \Delta n_{\beta}=\left|n_{\gamma}-n_{\alpha}\right| \approx \Delta n_{c}, \Delta n_{\gamma}=$ $\left|n_{\alpha}-n_{\beta}\right|$, respectively. The linear birefringence changes were measured using the rotating-analyser modulation method [9]. A $1 \mathrm{~mW} \mathrm{He} / \mathrm{Ne}$ laser served as a light source $(\lambda=632.8 \mathrm{~nm})$. In our method the light intensity measured has an amplitude proportional to $\sin \varphi$ ( $\varphi$ is the phase shift introduced by the optical retardation: $\varphi=(2 \pi / \lambda) \Delta n$ t, for wavelength $\lambda$, birefringence $\Delta n$, and thickness $t)$. The accuracy of this method appears to be better than $10^{-6}$. The cooling and heating rate used in the phase transition region was $0.05 \mathrm{~K} / \mathrm{min}$.

\section{Results and conclusions}

\subsection{The high temperature phase transition}

In Fig. 2a we present the temperature dependence of the linear birefringence changes $\Delta\left|\Delta n_{\beta}\right|$ and $\delta\left(\Delta n_{\beta}\right)$ for this phase transition in the BPI crystal. The temperature dependence of $\Delta\left|\Delta n_{\gamma}\right|$ and $\delta\left(\Delta n_{\gamma}\right)$ is shown in Fig. 3a. The linear birefringence increments $\delta\left(\Delta n_{i}\right)$ induced by the phase transition were obtained by substraction of the high temperature part from the temperature dependence 

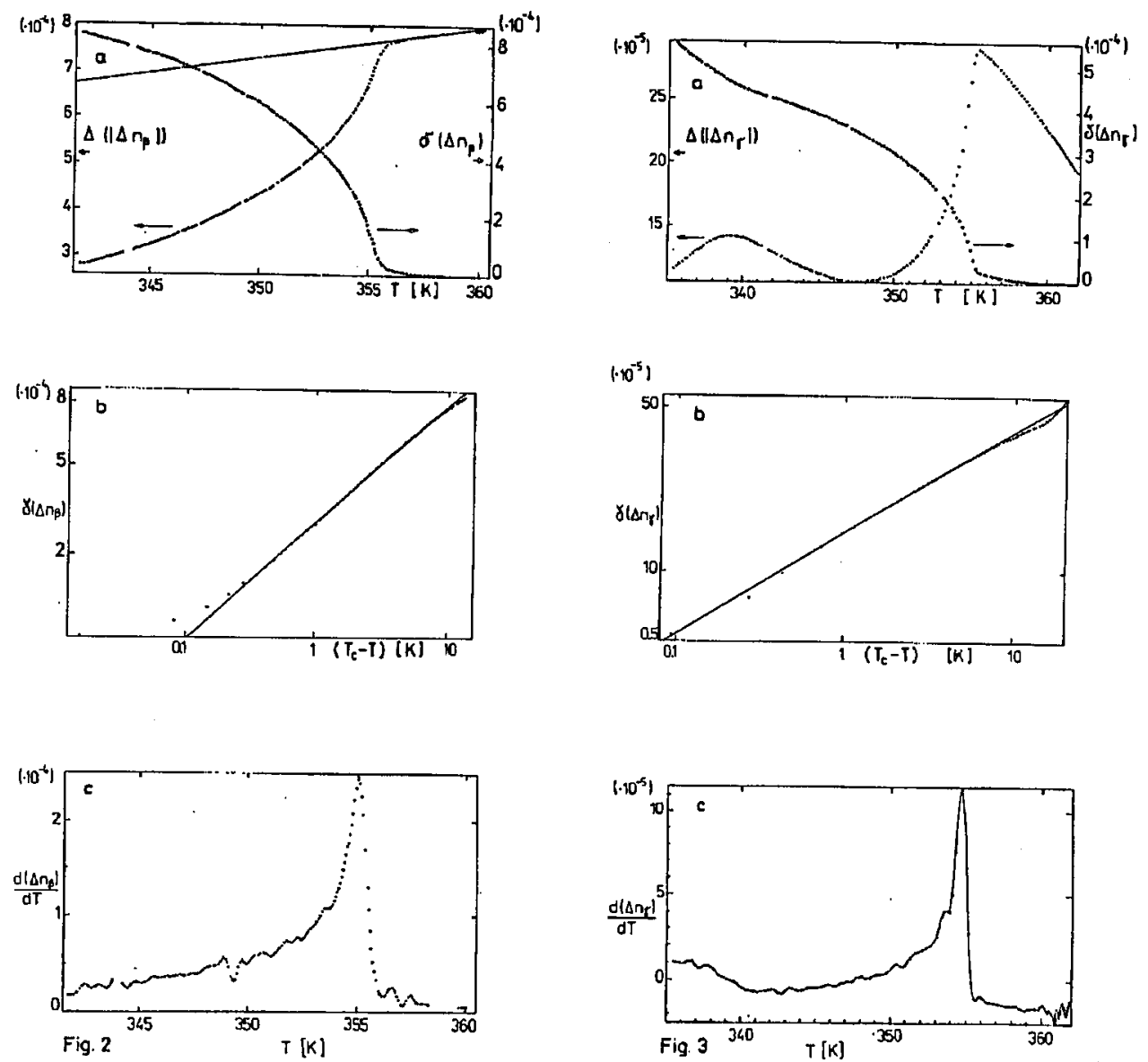

Fig. 2. Temperature dependence of $\Delta\left|\Delta n_{\beta}\right|$ and the linear birefringence increment $\delta\left(\Delta n_{\beta}\right)$ in the high temperature phase transition region $(\mathrm{a}) ; \log -\log$ plot of the $\delta\left(\Delta n_{\beta}\right)$ versus $T-T_{\mathrm{C}_{1}}, T_{\mathrm{C}_{1}}=355.15 \mathrm{~K}, 2 \beta=0.419 \pm 0.001$ (b); temperature derivative $\mathrm{d}\left(\left|\Delta n_{\beta}\right|\right) / \mathrm{d} T$ in the phase transition region (c).

Fig. 3. Temperature dependence of $\Delta\left|\Delta n_{\gamma}\right|$ and the linear birefringence increment $\delta\left(\Delta n_{\gamma}\right)$ in the high temperature plase transition region (a); log-log plot of the $\delta\left(\Delta n_{\gamma}\right)$ versus $T-T_{\mathrm{C}_{1}},-T_{\mathrm{C}_{1}}=354.92 \mathrm{~K}, 2 \beta=0.430 \pm 0.002$ (b); temperature derivalive $\mathrm{d}\left(\left|\Delta n_{\gamma}\right|\right) / \mathrm{d} T$ in the phase transition region (c).

of $\Delta\left|\Delta n_{i}\right|$. There was not any discontinuily in the $\Delta\left|\Delta n_{i}\right|(T)$ dependencies observed in the phase transition region. The distinct smearing $(3-5 \mathrm{~K})$ of $\delta\left(\Delta n_{i}\right)(T)$ at about $T_{\mathrm{C}_{1}}$ is probably caused by the order parameter fluctuations [8]. The critical exponent $\beta$ was calculated from the slope of $\ln \delta(\Delta n)$ versus $\ln \left(T-T_{\mathrm{C}}\right)$ dependencies, Figs. $2 \mathrm{~b}$ and $3 \mathrm{~b}$. The temperature derivatives $\mathrm{d}(|\Delta n(T)|) / \mathrm{d} T$ at the phase transition region are shown in Figs. $2 \mathrm{c}$ and $3 \mathrm{c}$. The high temperature structural phase transition $\left(P 12_{1} / m 1 \Rightarrow P 12_{1} / c 1\right)$ is accompanied with the doubling of the unit cell along the $c$-axis. By analogy to the BP crystal the meaning of the order parameter of this phase transition is a rotation of the betaine and/or 
phosphites groups around an axis lying in the mirror ac plane. This rotational mode with $k=c^{*} / 2$ transforms according to the representation $B_{g}$ of $P 2_{1} / m$ and the order-disorder transition can be classified as antiferrodistortive and improper ferroelastic with characteristic elastic anomalies [10, 11]. According to Dvorak [12] the free energy can be expanded as

$$
F=(1 / 2) a \eta^{2}+(1 / 4) b \eta^{4}+(1 / 6) c \eta^{6}+\gamma_{1} \eta^{2} u_{x x}+\gamma_{2} \eta^{2} u_{y y}+\gamma_{3} \eta^{2} u_{z z}+\gamma_{4} \eta^{2} u_{x z},
$$

where $u_{i i}$ are the spontaneous deformations. From this it follows that $u_{i i} \propto \eta^{2}$. These $u_{i i}$ components are not symmetry breaking components but nevertheless additional parts are induced by the coupling with $\eta$. The critical index determination is slightly difficult because of the significant blurring of the transition in the phase transition region. The slope of the $\log -\log$ plots of $\delta(\Delta n)$ vs. $\left(T_{\mathrm{C}}-T\right)$ is about $0.415-0.430$ and assuming $\delta(\Delta n) \propto \eta^{2 \beta}$, the order parameter exponent $\beta$ is about $0.210-0.220$. The very similar phase transition in the BP crystal is relatively close to the tricritical point [13]. The critical properties of the three-dimensional ANNI model studied by the Monte Carlo analyses based on coarse graining of single distribution function lead to the critical exponent in the vicinity of the Lifshitz point $\beta_{\mathrm{L}}=0.19 \pm 0.02$ [14]. We noticed that the high temperature phase transition was easily detected for the $\beta$ - and $\gamma$-cut, but for the $\alpha$-cut was rather hardly observable. The linear birefringence changes induced by the low temperature ferroelectric phase transition were more pronounced for the $\alpha$ - and $\gamma$-cut, whereas for the $\beta$-cut were small and difficult to detect. The comparative plot of derivatives $\mathrm{d}\left(\left|\Delta n_{i}\right|\right) / \mathrm{d} T$ is presented in Fig. 4.

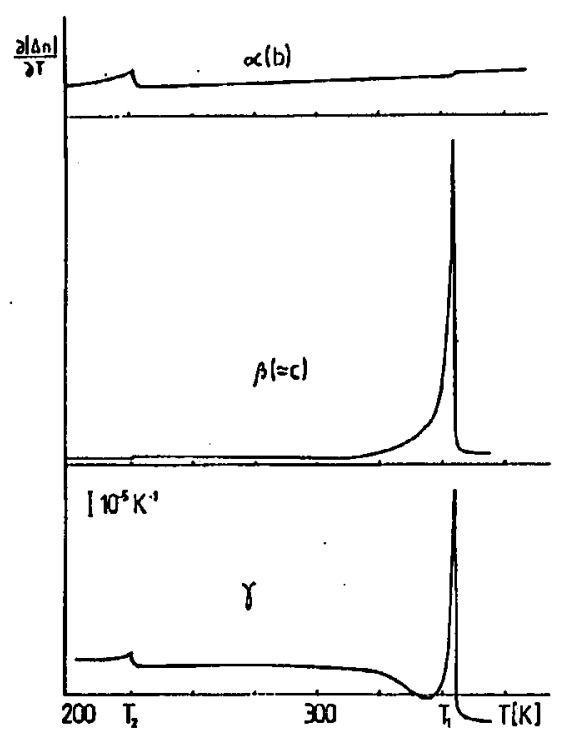

Fig. 4. Comparative plot of derivatives $\mathrm{d}\left(\left|\Delta n_{i}\right|\right) / \mathrm{d} T$ for both transitions. 


\subsection{The low temperature phase transilion}

In Fig. 5a, the linear birefringence changes $\Delta\left|\Delta n_{\alpha}\right|(T)$ and the linear birefringence increment $\delta\left(\Delta n_{\alpha}\right)(T)$ are presented. The linear birefringence increments $\delta\left(\Delta n_{\beta}\right)(T)$ and $\delta\left(\Delta n_{\gamma}\right)(T)$ are shown in Figs. $6 \mathrm{a}$ and $7 \mathrm{a}$, respectively. The critical exponent determined from the $\ln \left(\delta\left(\Delta n_{i}\right)\right)$ vs. $\ln \left(T_{\mathrm{C}}-T\right)$ plots is very close to 0.50
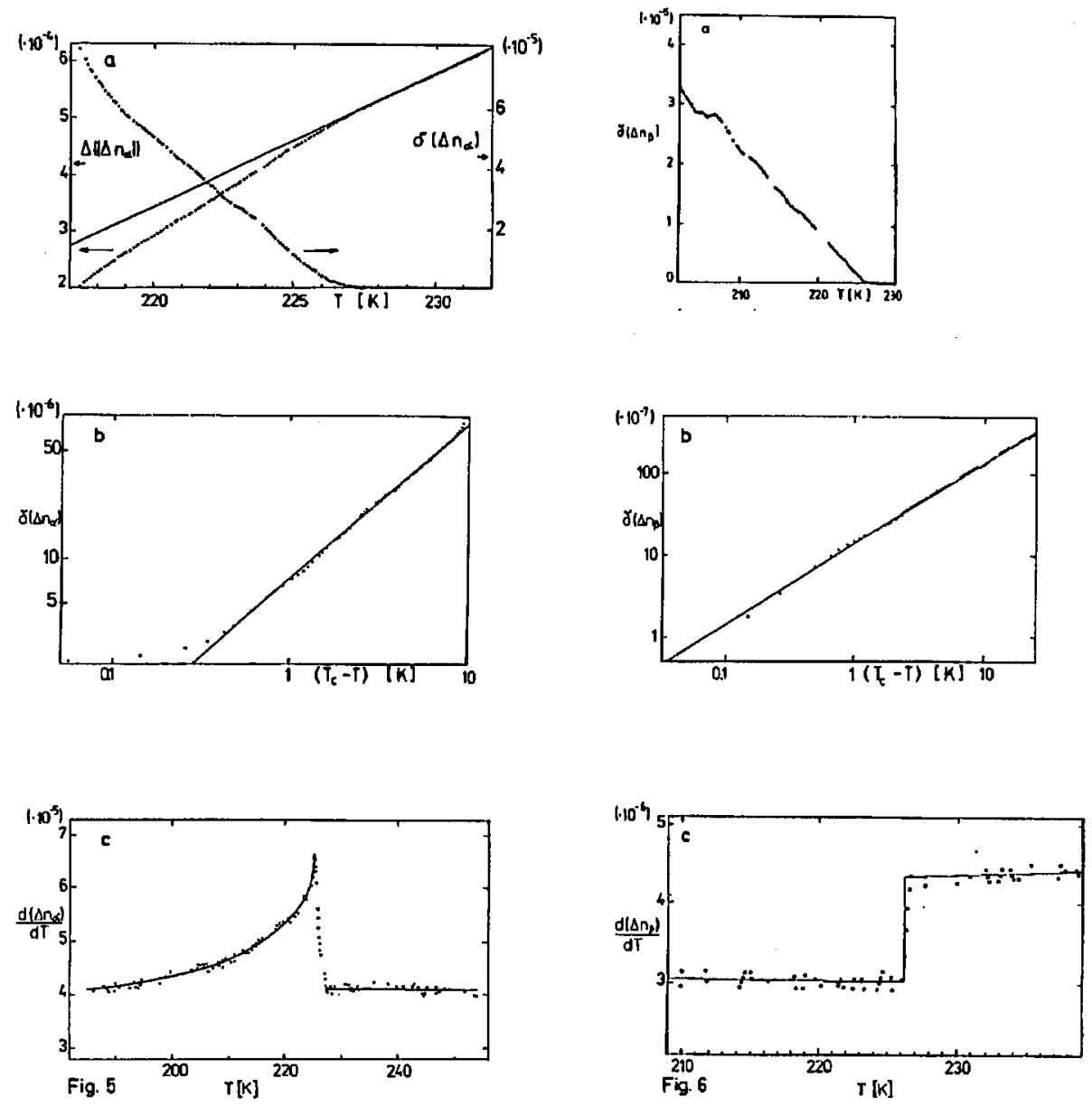

Fig. 5. Temperature dependence of $\Delta\left|\Delta n_{\alpha}\right|$ and the linear birefringence increment $\delta\left(\Delta n_{\alpha}\right)$ in the ferroelectric phase transition region (a); log-log plot of the $\delta\left(\Delta n_{\alpha}\right)$ versus $T-T_{\mathrm{C}_{2}}, T_{\mathrm{C}_{2}}=226.61 \mathrm{~K}, 2 \beta=1.031 \pm 0.004(\mathrm{~b})$; temperature derivative $\mathrm{d}\left(\left|\Delta n_{\alpha}\right|\right) / \mathrm{d} T$ in the phase transition region (c).

Fig. 6. Temperature dependence of the linear birefringence increment $\delta\left(\Delta n_{\beta}\right)$ in the ferroelectric phase transition region (a); log-log plot of the $\delta\left(\Delta n_{\beta}\right)$ versus $T-T_{\mathrm{C}_{2}}$, $T_{\mathrm{C}_{2}}=226.26 \mathrm{~K}, 2 \beta=0.994 \pm 0.002(\mathrm{~b}) ;$ temperature derivative $\mathrm{d}\left(\left|\Delta n_{\beta}\right|\right) / \mathrm{d} T$ ill the phasc transition region (c). 

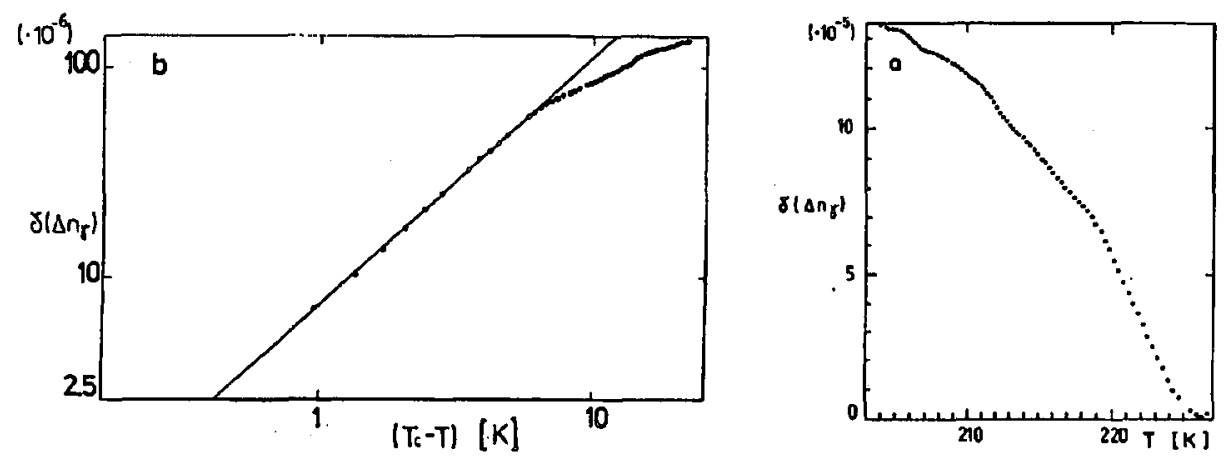

Fig. 7. Temperature dependence of the linear birefringence increment $\delta\left(\Delta n_{\gamma}\right)$ in the ferroelectric phase transition region (a); log-log plot of the $\delta\left(\Delta n_{\gamma}\right)$ versus $T-T_{\mathrm{C}_{2}}$, $T_{\mathrm{C}_{2}}=225.65 \mathrm{~K}, 2 \beta=1.102 \pm 0.006(\mathrm{~b})$.

(Figs. 5b, 6b, and $7 \mathrm{~b}$ ). The linear birefringence derivatives are shown in Figs. 5c and $6 \mathrm{c}$. A square of the polarization along the $b$-axis calculated by integrating the pyroelectric current is in fact linear in the temperature range of about $9 \mathrm{~K}$ below $T_{\mathrm{C}_{2}}$ [15]. The transition is of the second order and of the order-disorder type. The ferroelectric phase transition temperature reported differs significantly: 208-226 K, and is probably correlated with crystal quality [16]. The low temperature structure is not fully described because of the strong pseudosymmetry [2]. Deviations of the hydrogen atoms from their positions on the $y$-axis lead to a vanishing of the c-glide-mirror plane. The anomaly observed in the dielectric measurements at about $177-200 \mathrm{~K}$ has not been reproducible during the linear birefringence measurements and will be presented and described in the next paper.

\section{Acknowledgment}

This work was supported by the Research Project No. 201289101.

\section{References}

[1] G. Schaack, Ferroelectrics 104, 147 (1990).

[2] Th. Narz, Doktorarbeit, Universität Köln, Köln 1989; see also Ref. [16], p. 40.

[3] W. Schildkamp, J. Spilker, Z. Kristallogr. 168, 159 (1984).

[4] J. Albers, A. Klöpperpieper, H.J. Rother, S. Haussühl, Ferroelectrics 81, 27 (1988).

[5] J. Fousek, J. Petzelt, Phys. Status Solidi A 55, 11 (1979).

[6] J. Kroupa, J. Albers, N.R. Ivanov, Ferroelectrics 105, 345 (1990).

[7] J. Kroupa, J. Albers, Ferroeleclrics 108, 341 (1990).

[8] J. Kroupa, Ferroelectrics 124, 145 (1991).

[9] I.G. Wood, A.M. Glazer, J. Appl. Cryst. 13, 217 (1980).

[10] V. Dvorak, Ferroelectrics 104, 135 (1990).

[11] M. Maeda, J. Phys. Soc. Jpn. 57, 3059 (1988). 
[12] V. Dvorak, private communication.

[13] J. Albers, E.V. Balashova, A. Klöpperpieper, V.V. Lemanov, H.E. Müser, A.B. Sherman, Ferroelectrics 108, 357 (1990).

[14] K. Kaski, W. Selke, Phys. Rev. B 31, 5, 3128 (1985).

[15] S. Dacko, Z. Czapla, R. Sobiestianskas, in: Proc. 20th Spring Conf. on Ferroclectricity, Eds. G. Schmidt, E. Sorge, Marthin-Luther-University, Halle 1992, p. 87.

[16] S. Launer, Diplomarbeit, Universität Würzburg, Würzburg 1991, p. 42. 